\title{
Application of card sorting approach to classify human factors of past maritime accidents
}

\author{
Beatriz Navas de Maya ${ }^{\mathrm{a}}$, Hassan Khalid $^{\mathrm{b}}$ and Rafet Emek Kurt ${ }^{\mathrm{a} *}$ \\ rafet.kurt@strath.ac.uk \\ ${ }^{a}$ University of Strathclyde, 100 Montrose St, Glasgow G4 OLZ, United Kingdom \\ ${ }^{b}$ National University of Science and Technology, PNEC, Karachi, Pakistan
}

\begin{abstract}
Maritime accidents are complex processes in which many factors are involved and contribute to accident development. In order to capture underlying factors in accidents, countries adapted an accident investigation system with the aim of learning from these rare events and prevent similar occurrences in the future. Often these accident investigation reports are converted into databases, which lack a concise and user-friendly classification system, as a result there are a lot of inadequacies in data-collection and tagging procedures. Therefore, the authors propose to apply an approach to classify human factors (HFs) appeared in past maritime accidents, aiming to develop a set of HFs categories which can be used for accidents learning. For this purpose, an accident database was obtained and a two-stage approach is adapted to conduct analysis: first, an open card-sorting case study is organised to group the HFs extracted from an historical accident database. Second, a hybrid card-sorting method is utilized to fully achieve the classification of HFs. Our study revealed issues where HFs are weakly defined and similar factors are duplicated by investigators who populate the database. High level categories were developed and presented which covers great majority of HFs concerns involved in accidents.
\end{abstract}

Keywords: maritime accidents; human factors; shipping; card-sorting; safety

\section{Introduction}

Review of available literature reveals that human factors (HFs) have always had a high contribution into past accidents in diverse strategic sectors (i.e. aviation or nuclear) (Azadeh \& Zarrin, 2016). Maritime industry is no exception; various authors have studied the role of HFs in past accidents. They believe that around $80 \%$ of maritime accidents are attributable to human error (Graziano, Teixeira, \& Guedes Soares, 2016; Navas de Maya, Kurt, \& Turan, 2018; Turan et al., 2016). Some studies have found human-factor contributing as high as $96 \%$ (Rothblum, 2000). Despite all research efforts of the past several decades to address HFs efficiently, it is still very difficult to precisely specify HFs responsible for maritime accidents (Jeong et al., 2016).

With the aim to better understand and address the HFs, one of the most significant measures that the maritime sector has implemented is the investigation of ships' accidents by expert, and by extension, the communication of findings to the authorities. This procedure is also encouraged and addressed by the international legislations (e.g. UNCLOS or SOLAS) (Kececi \& Arslan, 2017). Yet, there is a lack of consensus between maritime organizations responsible of accidents investigations. Each organization has a unique data-collection procedure and 
nomenclature to define accident-contributing factors, which makes it extremely challenging to find common human factors across diverse accident databases. In order to understand and analyse accidents meaningfully, it is essential that the information regarding the sequence of events and related HFs is available. Unfortunately, accident databases for the maritime domain have not reached to their full potential in this respect. HFs identified in most accidents, usually, do not match the reality of the accident, as they are inconsistently recorded and lack a clear description. This is mainly attributable to the absence of a well-defined human-factors structure, which could allow accident investigators to relate each maritime accident to the most likely HFs. Such as, structure should also allow us to identify the HFs having high contribution into accidents. So that efforts and resources may be invested to develop strategies to mitigate such HFs to improve overall maritime safety.

This paper collects and analyses information about HFs considered to be involved in past maritime accidents from MAIB historical accident database. Above mentioned accident database investigates marine accidents involving UK vessels worldwide and all vessels operating in UK territorial waters, covering a period from 1990 to 2016. Thus, aforementioned database follows the European Marine Casualty Information Platform (EMCIP) taxonomy (EMSA, 2016), which in line with the Event and Contributing Factors Analysis (ECFA) model (Buys \& Clark, 1978). Within EMCIP taxonomy, accidents are investigated depending upon their severity, following a process in which certain human contributing factors listed within EMCIP taxonomy are identified as responsible for the accident. Nevertheless, above-mentioned human contributing factors often lack a clear and concise definition; hence, accident investigators might wrongly assign them when selecting the most influential human contributing factors in an accident scenario. Therefore, the purpose of this research is to understand HFs' structure and nomenclature of MAIB database, so as to develop a set of more generic and better-defined human-factors groups by applying card-sorting technique. Experts of accident investigations, HFs and ship operations were invited to participate in this study to achieve afore-mentioned human-factor classification.

The paper begins with a review of previous studies on HF classification and their contribution into maritime accidents followed by a review of card-sorting method, encompassing its definition, application and available techniques to analyse results. In Section 2, the methodology is explained. Results and discussion are outlined in Section 3 and 4 respectively. Finally, Section 5 concludes the paper.

\section{Literature review}

\section{1- Previous studies on human-factor classification and contribution into accidents in the maritime sector}

In recent years, various researchers have conducted numerous studies to identify and successfully classify and evaluate the contribution of HFs into past maritime accidents. There appears to be three major approaches. Firstly and perhaps the most commonly applied techniques to analyse and classify HFs is the Human Factor Analysis and Classification System (HFACS)that aims to identify latent human errors. It has been successfully applied for accident analysis in numerous fields (Luo \& Shin, 2016). Secondly, Human Reliability Assessments (HRAs) is employed to predict human errors, while allowing the adoption of human element within the risk framework (Kirwan, 1996; Smith, Veitch, Khan, \& Taylor, 2017). Lastly, a less heuristics and more data intensive approach is the statistical analysis. 
HFACS method was originally designed for military aviation with two purposes. Firstly, to facilitate HFs accident investigation and secondly, to underline the causes of human error and analyse them. HFACS has been successfully applied to other strategic industries such as civil aviation, railway, mining (Chauvin, Lardjane, Morel, Clostermann, \& Langard, 2013) and maritime (Luo \& Shin, 2016). Chauvin et al. (2013) analysed maritime accidents by applying the HFACS method, identifying Situational Awareness (SA) and a deficit of attention as significant HFs leading to collision accidents. Yıldırım, Başar, and Uğurlu (2017) assessed grounding accidents by applying HFACS, highlighting that insufficient communication or a lack of procedures strongly related to grounding accidents. HFACS-based techniques have also been developed and applied to specific accidentscenarios in the maritime sector. For example, Chen et al. (2013) developed and applied an HFACS-based method specifically designed for the maritime sector (i.e. HFACS-MA). Moreover, Celik and Cebi (2009) proposed to create an HFACS based on a fuzzy analytical hierarchy process, and Schröder-Hinrichs, Baldauf, and Ghirxi (2011) adapted HFACS to the machinery spaces on ships (i.e. HFACS-MSS). Although, HFACS has a strong theoretical foundation (i.e. it is based on Reason's ideas and theory), it presents some limitations. HFACS has industry restrictions. As it was designed to analyse the causes of aviation accidents, some categories within this model are not applicable to other sectors. Hence, HFACS presents a remarkable lack of versatility. For example, psychological factors can be identified by interviewing relevant personnel when applying HFACS, however, these will be limited due to the subjectivity of interviews' results (Fu, Cao, Zhou, \& Xiang, 2017). Moreover, HFACS limits the scope of investigation to a predefined taxonomy and organization, hence, it will be altered in each accident outcome or scenario being modelled.

Apparently, HRAs can be divided into two different generations. First generation associates the concept of human error with people's deficiencies. On the other hand, second generation of HRAs is characterized by approaching human behaviour to risk analysis, requiring a more complex and integrating model validation and Performance Shaping Factors (PSFs) (Yang, Bonsall, Wall, Wang, \& Usman, 2013). Within the scope of the maritime sector, the second generation CREAM method is a widely used HRAs. For instance, Zhou et al. (2017) applied an enhanced CREAM method by incorporating stakeholders-graded protocols to a tanker shipping safety. Ung (2015) developed a weighted CREAM model to analyse maritime human reliability. Recently, Zhang, He, Chen, Chu, and Fan (2019) applied a Predictive Mean Vote (PMV)-CREAM to perform a dynamic human reliability assessment to manned submersibles. Ung (2019) applied fault tree analysis and modified fuzzy Bayesian Network-based CREAM to evaluate the human error contribution to oil tankers. Majority of the aforementioned HRA applications use expert judgment, as contribution of a human element to an accident is difficult to quantify numerically. However, incorporation of expert judgment makes the results somewhat subjective. Alike HFACS, HRAs are designed for a specific sector or scenario. This limitation does not allow creating a generic model, which could apply to all maritime accident. The necessity to model a new scenario for each case study is time and cost consuming. Thus, there is a lack of a general purpose technique, which could be applied to any accident scenario, to identify and quantify the main HFs contributing toward the maritime accident.

Several researchers have used statistical analyses to identify accident contributing-factors (Bye \& Aalberg, 2018; Eliopoulou, Papanikolaou, \& Voulgarellis, 2016; Navas de Maya, Ahn, \& Kurt, 2019; Papanikolaou, Bitha, Eliopoulou, Ventikos, \& Engineering, 2014; Ventikos, Papanikolaou, Louzis, \& Koimtzoglou, 2018; Y1ldırım et 
al., 2017). However, aforementioned efforts of statistically modelling the relationship between contributors and accidental outcomes have been very challenging due to data type and inconsistency in its collection procedures.

\section{2- Review on card-sorting method}

Card-sorting method is a qualitative technique that was developed in 1980s (Tullis, 1985). However, it was not until early 2000s that it got popular as a user experience design method (Hudson, 2012; Sinha \& Boutelle, 2004). Since "card sort" technique is around for quite some time (Rugg \& Petre, 2007), it has several definitions. According to Paul (2014), card-sorting is a method to elicit people's underlying mental models about a conceptual domain. Faiks and Hyland (2000) define it as a qualitative technique, where participants sort cards into piles according to their own perceptions. Card-sorting method is easy to replicate (Faiks \& Hyland, 2000), and allows saving of resources (e.g. time, funds and a large number of participants) (Nicholson, 2016). It has become a popular information architecture method in the past decade (Paul, 2014).

Traditional card-sorting techniques involve sorting a set of pictures, objects or labelled cards into distinct categories (Faiks \& Hyland, 2000; Fincher \& Tenenberg, 2005; Rugg \& Petre, 2007). A card-sorting study may be conducted in various ways. However, the general methodology is similar for all cases (Paul, 2014). First, a set of concepts (i.e. cards), which represent a research domain are created. Second, participants are asked to sort these cards into different groups based on their own interpretation. Groups may be predefined by the researchers (i.e. a closed card-sort study), or they may be created by the participants (i.e. open card-sort study). Alternatively, a combination of both is also possible; where some groups are predefined but participants are allowed to create additional groups (i.e. a hybrid case study). Third, participants name the groups based on the topic they represent (only applicable to open or hybrid card-sorting case studies) (Nicholson, 2016; Paul, 2014).

Being easy to conduct, card-sorting has been successfully applied in various domains with diverse objectives. For instance, Eppler \& Platts (Eppler \& Platts, 2007) used it to explore people's mental models of classifying visualization methods. It has also been applied for task analysis of geo-visualization tools and interactions (Lloyd, Dykes, \& Radburn, 2008). Briefly, card-sorting has been used as a tool in marketing, computer science, and psychological investigations (Duncan, 2012). Dimensional Change Card Sort (DCCS) and Wisconsin Card Sort (WCS) are widely used in neuroscience, neuropsychiatry and psychology, and the Pediatric Activity Card Sort (PACS) is commonly used as an occupation based assessment tool (Bialystok \& Martin, 2004; Haaland, Vranes, Goodwin, \& Garry, 1987; Laws, 1999; Zelazo, 2006).

As such, card-sorting data can be analysed using both, qualitative and quantitative methods (Paul, 2014). However, the most common methods to analyse card-sorting data are qualitative in nature (Fincher \& Tenenberg, 2005; Righi et al., 2013).Qualitative methods strongly rely on designer's intuition and interpretation. A qualitative analysis is mainly focused on understanding the topics created by each participants, and the cards grouped under each topic. Traditional techniques include manually analyzing and understanding the agreement of group and topic naming, the meaning of the topics, the relationships between groups, the context of participant use, or the domain of use (Deibel, Anderson, \& Anderson, 2005; Paul, 2014; Sanders et al., 2005). One of the widely used qualitative techniques is topic normalization, which consists of merging similar participant-created groups (i.e. groups with a similar terminology) into a single topic for further analysis (Paul, 2014). However, qualitative analysis is a manual, time-consuming process that trades off analysis effort with a rich understanding of the data 
(Paul, 2014). On the other hand, quantitative analysis methods include descriptive and statistical techniques. Descriptive methods examine the shape of the data, such as the number of groups and number of cards within groups (Deibel et al., 2005), while statistical methods assess the significance of features within the data (Deibel et al., 2005; Sanders et al., 2005).

Card-sorting technique offers numerous advantages. To begin with, it is easy to conduct and repeat, as instructions are simple for the organizer and the participants. Also, the card-sorting sessions have minimal set up cost. It is a well-establish discipline amongst usability professionals in various domains (Duncan, 2012). More importantly, card-sorting procedure generally involves end users, which provide insight into users' mental models (Eppler \& Platts, 2007). Nevertheless, card-sorting method do have certain limitations. Firstly, it may produce varied results; hence, the card sort may fail to provide consistent results between participants, or the results may vary widely. In addition, the analysis of the results is time consuming. Although the setup of the card-sorting session can be achieved quickly, the data analysis stage can be extensive, complicated and time-consuming, particularly if the results are inconsistent. Lastly, if the labels are not chosen in the context of a specific study, the ability of the labels to represent meaning is reduced or eliminated.

\section{Methodology}

As mentioned in the previous section, establishing improved human-factors structure would facilitate accident investigators to identify and assign HFs involved in maritime accidents, more efficiently. Hence, classifying HFs from an accident database into generic, yet clearly defined HFs groups is the objective of this study. Firstly, a historical accident database was obtained and analyzed to identify the HFs reported to be likely contributors in past accidents. Secondly, an open card-sorting case study was created to group the aforesaid HFs into more generic and better-defined new HF groups. Special attention was given to the selection of participants so as to obtain more reliable results. Thirdly, the results were analyzed qualitatively (i.e. topic normalization and expert opinion analysis were conducted) and quantitatively (i.e. statistical analysis and co-occurrence analysis). A total of eleven HFs groups were created, where participants had successfully sorted the majority of initial HFs (78 out of 94 in number) from the accident database. Thereafter, a new hybrid card-sorting case study was created for the classification of unsorted HFs. The results of the study were analyzed by applying statistical techniques. In that, the proposed human-factors classification was compared with the results obtained from the co-occurrence analysis, aiming to re-define the classification and to validate the results. In addition, field experts' judgments were used to assess and fine-tune the newly proposed HFs' classification. Figure 1 shows the procedure adopted to develop the new human-factor classification structure proposed within this study. 


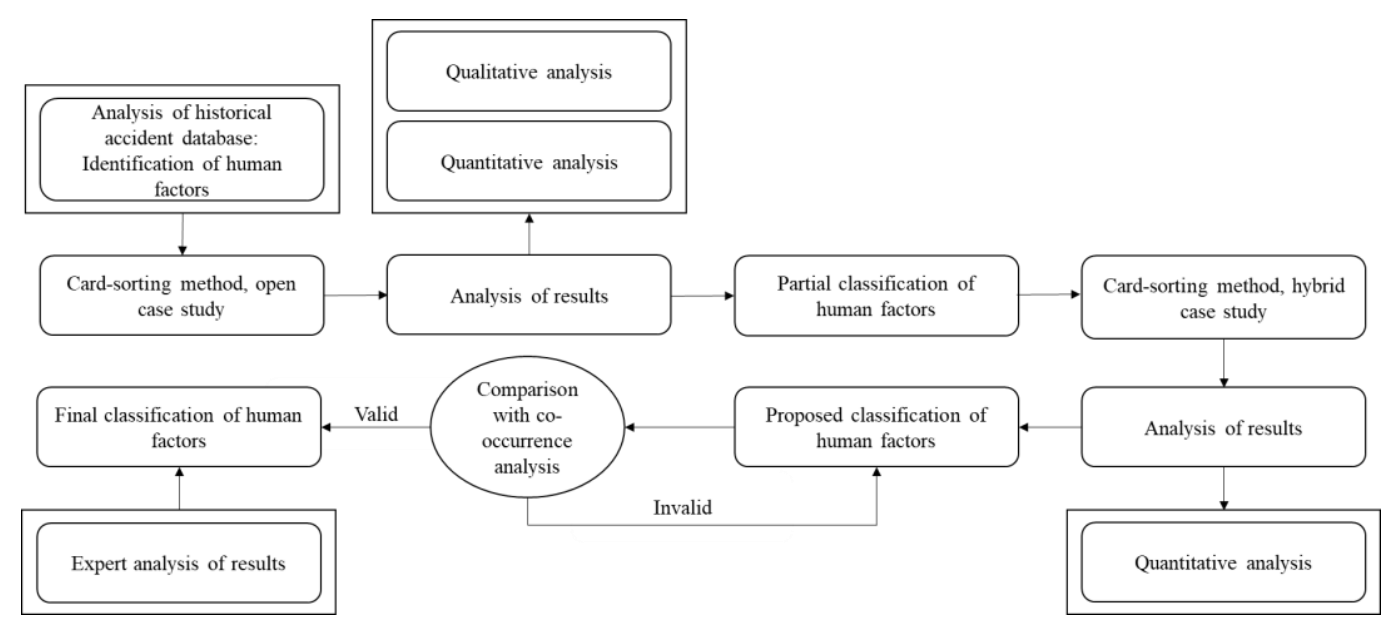

Figure 1. Classification of human factors flowchart

Finally, the reliability and the validity of this study were assessed in the discussion section by applying the triangulation method, which can be of three types as follows: methodological, expert and theoretical. The methodological triangulation relies on using several instruments to get the information, aiming to obtain similar results through different ways. The expert triangulation relies on asking certain experts about the suitability of the research methodology. In addition, the theoretical triangulation consists on comparing the qualitative results with previous literature (Flick, 2018).

\section{Results}

\section{1- Data selection, identification of human factors}

In order to fulfil the aims of this paper, an accident database, which registers marine accidents involving UK vessels worldwide and all vessels operating in UK territorial waters between 2011 and 2016 was obtained from Marine Accident Investigation Branch (MAIB) and analyzed. This period was selected as MAIB started recording HFs using European Marine Casualty Information Platform (EMCIP) nomenclature in 2011. The database was found to contain ninety-four HFs, likely to be responsible for maritime accidents. A list of these HFs is displayed in Table 1.

Table 1. Initial list of human factors identified from the MAIB accident database

\begin{tabular}{|c|c|c|c|c|c|}
\hline No & Human factor & No & Human factor & No & Human factor \\
\hline 1 & Anthropometric factors, dimensions & 33 & Inadequate promotion of Safety & 65 & $\begin{array}{l}\text { LTA* physical/ } \\
\text { physiological capability }\end{array}$ \\
\hline 2 & Audit & 34 & $\begin{array}{l}\text { Inadequate standards or } \\
\text { specifications }\end{array}$ & 66 & LTA* planning \\
\hline 3 & Checks & 35 & Inadequate testing & 67 & $\begin{array}{l}\text { LTA* Safety plan and } \\
\text { program }\end{array}$ \\
\hline 4 & $\begin{array}{l}\text { Conflicting orders, cross-pressure } \\
\text { (ex. master's standing orders) }\end{array}$ & 36 & Inadequate training program & 68 & $\begin{array}{l}\text { LTA* System review and } \\
\text { evaluation }\end{array}$ \\
\hline 5 & Contingency plans not updated & 37 & Inadequate work methods & 69 & Management training \\
\hline 6 & Cowboy attitudes, horseplay & 38 & $\begin{array}{l}\text { Inadequate work preparation } \\
\text { (ex. passage plan) }\end{array}$ & 70 & $\begin{array}{l}\text { No review or critical tasks / } \\
\text { operations }\end{array}$ \\
\hline 7 & Crisis handling & 39 & Inappropriate peer pressure & 71 & $\begin{array}{l}\text { Person-to-person conflict / } \\
\text { animosity }\end{array}$ \\
\hline 8 & $\begin{array}{l}\text { Cross-pressure from schedule \& } \\
\text { economy }\end{array}$ & 40 & Inappropriate regulations & 72 & $\begin{array}{l}\text { Pressure to keep schedule } \\
\text { and costs }\end{array}$ \\
\hline 9 & Design (DESIGN) & 41 & Inspection & 73 & Regulation \\
\hline
\end{tabular}




\begin{tabular}{|c|c|c|c|c|c|}
\hline No & Human factor & No & Human factor & No & Human factor \\
\hline 10 & Design error & 42 & $\begin{array}{l}\text { Lack of communication \& } \\
\text { coordination }\end{array}$ & 74 & Regulatory procedures \\
\hline 11 & $\begin{array}{l}\text { Deviation from } \\
\text { standards/specifications }\end{array}$ & 43 & Lack of co-ordination of tasks & 75 & Regulatory standards \\
\hline 12 & Display design, controls & 44 & $\begin{array}{l}\text { Lack of information, } \\
\text { inadequately presented } \\
\text { information }\end{array}$ & 76 & Resistance to change \\
\hline 13 & Emergency plans & 45 & Lack of knowledge & 77 & Restricted fairway \\
\hline 14 & Emergency procedures & 46 & Lack of leadership & 78 & $\begin{array}{l}\text { Right tools and equipment } \\
\text { unavailable }\end{array}$ \\
\hline 15 & Emergency training program & 47 & Lack of maintenance & 79 & $\begin{array}{l}\text { Safety awareness, cutting } \\
\text { corners }\end{array}$ \\
\hline 16 & $\begin{array}{l}\text { Expectations of supervisor is } \\
\text { unclear }\end{array}$ & 48 & Lack of motivation/morale & 80 & Sea motion \\
\hline 17 & Failure not detected during IMR* & 49 & Lack of priority to IMR* & 81 & Selection/training of officers \\
\hline 18 & Follow-up of non-conformities, & 50 & Lack of resources & 82 & $\begin{array}{l}\text { Social \& cultural barriers \& } \\
\text { conflicts }\end{array}$ \\
\hline 19 & Frequent change of watch schedule & 51 & $\begin{array}{l}\text { Lack of responsibility for own } \\
\text { job }\end{array}$ & 83 & Supervision (SUPER) \\
\hline 20 & Hazardous/ messy workplace & 52 & Lack of skill & 84 & Supervisors not in touch \\
\hline 21 & Health control of personnel & 53 & Lack of warning systems & 85 & Surveillance \\
\hline 22 & Hiring and selection policy & 54 & $\begin{array}{l}\text { Lacks initiative to deal with } \\
\text { emergencies }\end{array}$ & 86 & $\begin{array}{l}\text { Too high work load / low } \\
\text { work load }\end{array}$ \\
\hline 23 & Idleness, waiting & 55 & Language problem & 87 & $\begin{array}{l}\text { Too low visibility for } \\
\text { observation }\end{array}$ \\
\hline 24 & $\begin{array}{l}\text { Improper performance of } \\
\text { maintenance/ repair }\end{array}$ & 56 & Lifesaving equipment & 88 & $\begin{array}{l}\text { Traffic density hinders vessel } \\
\text { control }\end{array}$ \\
\hline 25 & Improper supervisory example & 57 & $\begin{array}{l}\text { Long working periods, much } \\
\text { overtime }\end{array}$ & 89 & Training ignored \\
\hline 26 & $\begin{array}{l}\text { Inadequate briefing, instruction (ex. } \\
\text { passage briefing plan) }\end{array}$ & 58 & Low job satisfaction, monotony & 90 & $\begin{array}{l}\text { Unclear roles and } \\
\text { responsibility }\end{array}$ \\
\hline 27 & $\begin{array}{l}\text { Inadequate control of life saving } \\
\text { equipment }\end{array}$ & 59 & $\begin{array}{l}\text { LTA* assessment of needs and } \\
\text { risks }\end{array}$ & 91 & Use of wrong equipment \\
\hline 28 & Inadequate fighting equipment & 60 & $\begin{array}{l}\text { LTA* communication (oral, } \\
\text { written/read and visual) }\end{array}$ & 92 & Work instruction \\
\hline 29 & Inadequate illumination & 61 & LTA* design verification & 93 & Work place inspections \\
\hline 30 & Inadequate maintenance & 62 & $\begin{array}{l}\text { LTA* Formal safety } \\
\text { assessment, risk analysis }\end{array}$ & 94 & Wrong person assigned \\
\hline 31 & Inadequate manning & 63 & $\begin{array}{l}\text { LTA* medical services } \\
\text { provided }\end{array}$ & $*$ & $\begin{array}{l}\text { IMR (Inspection, } \\
\text { Maintenance and Repairs) }\end{array}$ \\
\hline 32 & $\begin{array}{l}\text { Inadequate procedures and check } \\
\text { lists (ship/port, maintenance, } \\
\text { company, emergency, other) }\end{array}$ & 64 & $\begin{array}{l}\text { LTA* mental and } \\
\text { psychological state }\end{array}$ & $*$ & LTA (Less than Adequate) \\
\hline
\end{tabular}

\section{2- Open card-sorting case study}

To begin with, an open card-sorting case study was initiated, to combine the HFs found in the accident database into new and improved HFs groups. Participants with experience in relevant fields for this study (e.g. HFs, accident investigation or ship operations) were invited with the aim to obtain reliable results. Aforementioned participants were selected from diverse geographical areas, to capture social and cultural differences in maritime operations and procedures. The card-sorting case study was held in two phases. In the first phase, a workshop was organized, which allowed to obtain two set of answers ("Participant1" and "Participant2"). In the second phase, an online open card-sorting case study was launched to reach experts with extensive experience of maritime accidents investigations and knowledge on its database. Resultantly, two additional set of answers were collected ("Participant3" and "Participant4"). 
Regarding the first phase, initially, twelve experts were invited to attend a half-day workshop. Skilled and experienced people were selected to increase reliability of the results. However, ten experts attended and contributed their expertise to the workshop. Figure 2 provides an insight into the participants' background. Some participants had experience in more than one field; therefore, Figure 2 shows both, the main area of expertise of each participant, and the accumulate experience of the participants (i.e. a participants with experience as a seafarer and academic experience is represented in both groups). When some experts are more credible than others, their contribution must be multiplied by a coefficient, $\mathrm{w}_{\mathrm{i}}$, before combining it with other experts' opinions, to improve reliability. Nevertheless, many authors in the literature have also defended the use of wi=1 (Taber, 1987) as there is no agreed standard-way of defining such weightings. Since, the participants of this study had relatively similar credentials and expertise areas, unitary weighting was used for each participant.

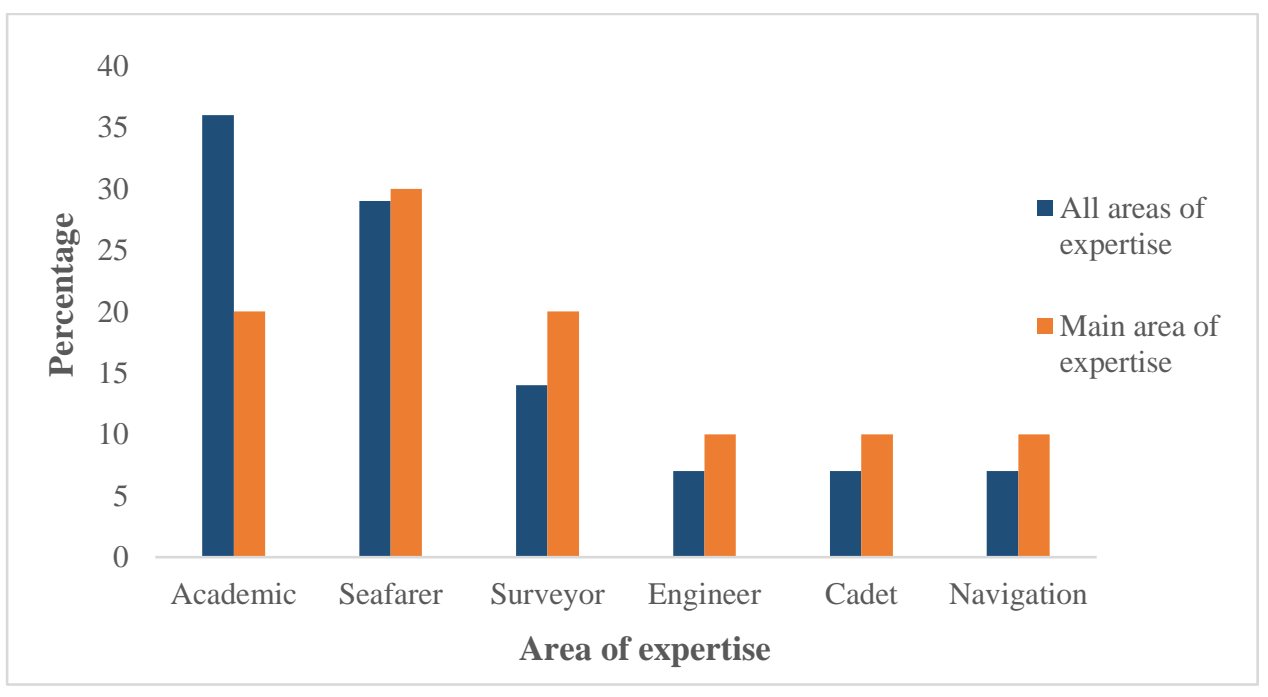

Figure 2. Background of participants

In this study, measures were taken to conduct the workshop ethically. Workshop attendance was voluntary and participants were informed that data would be analyzed for educational purposes. Thus, to maintain anonymity and confidentiality, names were not recorded, and only numbered tags identified participants.

The workshop was held at Strathclyde University on 21 March 2019. It consisted of three parts or steps. First, a description form was provided to each participant, highlighting the objectives and the structure of the workshop. Second, a presentation to the participants of the workshop objectives as well as a description of the card-sorting method was provided. This presentation was followed by a brief introduction of all experts to identify their main area of expertise. Based on the number and experience of each expert, two different groups ("Participant1" and "Participant2") were formed to sort the HFs found in accident database. Each group had a similar distribution in terms of background and work experience. The purpose of creating two different groups was to examine if groups of experts with similar expertise would classify HFs in a similar way. Finally, a feedback form was given to each participant to obtain further comments and recommendations.

Participants reviewed and sorted the ninety-four HFsinto improved categories using card-sorting technique. Although this activity could be carried out using a card-sorting software, it was decided to use physical cards. Use of real cards allowed each member to interact with the group, sharing his/ her ideas to classify each HF. A set of ninety-four human-factors descriptive cards were given to each group. These cards showed the number of each 
HF, and the title of each HF appeared in bold text. Finally, with the aim to provide additional information about each HF, especially for those cases where the HF is not self-explanatory, one or two examples were provided at the bottom of each card. These examples were extracted directly from accident investigators' comments recorded within the accident database. Figure 3 shows as an example the groups created by "Participant1" during the workshop session.

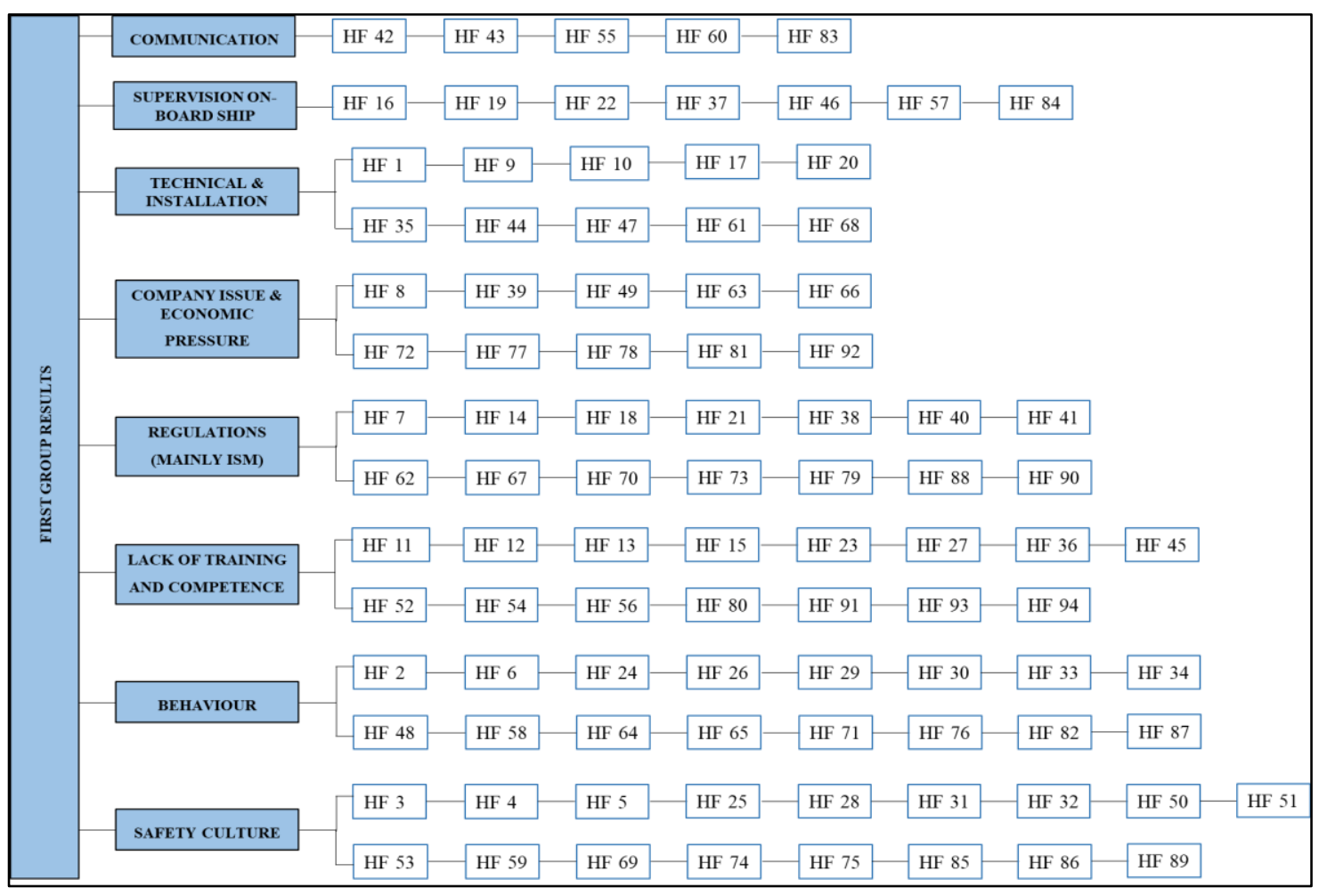

Figure 3. Groups created by "Participant1"

Regarding the second phase, an online open card-sorting case study was created using Optimal Sort software to remotely reach additional experts ("Participant3" and "Participant4"). Following the same structure as the workshop, the online case study included descriptions of ninety-four HFs from the historical accident database.

\section{3- Analysis of results}

Aiming to analyze the results obtained from the open card-sorting session, a set of qualitative analysis were conducted starting with topic normalization. In that experts reviewed the groups formed by the participants to merge the groups with similar terminology into single HF category. Moreover, expert opinion was sought to understand the data collected and analyze participants' answers. Statistical analysis were also conducted to assess the significance of features within the data. Co-occurrence analysis of the data was carried out to validate the pairs of cards that got sorted together during the open card session. In this study the co-occurrence analysis has been used to validate the proposed human-factor classification.

\section{Groups' normalization}

Topic normalization is one of the most common qualitative analysis techniques, which is widely applied to analyze the outcome of card-sorting sessions. The groups created by the four participants (i.e. teams) of the open case study were analyzed by means of this technique. Two experts on HFs compared the groups created within this 
case study and merged similar groups into common HF categories. To normalize groups, it was decided to consider those categories from the open case study in which at least two participants (i.e. teams) created a similar group. The only exemption to the previous requirement was the category "Substandard monitoring". It was agreed by the experts that a substandard monitoring category is very important and it should be retained, even if only one participant (team) created it. The list of the normalized groups proposed is shown in Table 2.

Table 2. Results from the open-case study. Normalised groups

\begin{tabular}{|c|c|c|c|}
\hline No & Group Name & No & Group Name \\
\hline 1 & Commercial pressure & 7 & Lack of training \\
\hline 2 & Effect of environmental and external factors & 8 & Safety culture \\
\hline 3 & $\begin{array}{l}\text { Improper design, installation and working } \\
\text { environment }\end{array}$ & 9 & $\begin{array}{l}\text { Safety management system: Inadequate procedures or } \\
\text { deviation from Standard Operating Procedure (SOP) }\end{array}$ \\
\hline 4 & Inadequate leadership and supervision & 10 & Safety management system: Substandard monitoring \\
\hline 5 & Lack of communication and coordination & 11 & Unprofessional behaviour \\
\hline 6 & Lack of, improper or late maintenance & & \\
\hline
\end{tabular}

\section{Statistical analysis}

Statistical analyses were carried out to identify how often given HFs got sorted in the same group. Table 3 provides those cards that were sorted together by at least half of the participants in the same group along with the statistical frequency and the normalized groups.

Table 3. Statistical analysis. Open card-sorting case study

\begin{tabular}{|c|c|c|c|c|c|c|c|c|}
\hline Card & Freq. & Group No & Card & Freq. & Group No & Card & Freq. & Group No \\
\hline 1 & 1.00 & Group 3 & 81 & 0.75 & Group 7 & 48 & 0.5 & Group 11 \\
\hline 9 & 1.00 & Group 3 & 84 & 0.75 & Group 4 & 50 & 0.5 & Group 8 \\
\hline 10 & 1.00 & Group 3 & 87 & 0.75 & Group 2 & 51 & 0.5 & Group 8 \\
\hline 15 & 1.00 & Group 7 & 88 & 0.75 & Group 2 & 53 & 0.5 & Group 3 \\
\hline 36 & 1.00 & Group 7 & 6 & 0.5 & Group 11 & 54 & 0.5 & Group 7 \\
\hline 8 & 0.75 & Group 1 & 14 & 0.5 & Group 9 & 55 & 0.5 & Group 5 \\
\hline 12 & 0.75 & Group 3 & 16 & 0.5 & Group 4 & 56 & 0.5 & Group 7 \\
\hline 29 & 0.75 & Group 3 & 18 & 0.5 & Group 9 & 58 & 0.5 & Group 11 \\
\hline 40 & 0.75 & Group 9 & 19 & 0.5 & Group 4 & 62 & 0.5 & Group 9 \\
\hline 42 & 0.75 & Group 5 & 23 & 0.5 & Group 7 & 67 & 0.5 & Group 9 \\
\hline 43 & 0.75 & Group 5 & 25 & 0.5 & Group 4 & 68 & 0.5 & Group 3 \\
\hline 45 & 0.75 & Group 7 & 27 & 0.5 & Group 7 & 70 & 0.5 & Group 9 \\
\hline 46 & 0.75 & Group 4 & 30 & 0.5 & Group 6 & 71 & 0.5 & Group 11 \\
\hline 52 & 0.75 & Group 7 & 33 & 0.5 & Group 11 & 73 & 0.5 & Group 9 \\
\hline 60 & 0.75 & Group 5 & 34 & 0.5 & Group 9 & 75 & 0.5 & Group 9 \\
\hline 61 & 0.75 & Group 3 & 37 & 0.5 & Group 4 & 76 & 0.5 & Group 11 \\
\hline 69 & 0.75 & Group 7 & 38 & 0.5 & Group 9 & 82 & 0.5 & Group 11 \\
\hline 72 & 0.75 & Group 1 & 39 & 0.5 & Group 1 & 83 & 0.5 & Group 4 \\
\hline 74 & 0.75 & Group 9 & 41 & 0.5 & Group 9 & 85 & 0.5 & Group 8 \\
\hline 77 & 0.75 & Group 2 & 44 & 0.5 & Group 3 & 89 & 0.5 & Group 8 \\
\hline 80 & 0.75 & Group 2 & 47 & 0.5 & Group 6 & 90 & 0.5 & Group 9 \\
\hline
\end{tabular}




\section{Expert analysis}

After the first quantitative analysis (i.e. statistical analysis) were conducted, the results of the open card-sorting case study were assessed by two experts in HFs in order to understand the data collected and to analyze participants' answers. The aim of this analysis was to group additional HFs that were not classified successfully by means of statistical analysis. This allowed to sort HF2, HF3, HF4, HF5, HF13, HF17, HF21, HF22, HF24, HF26, HF32, HF35, HF49, HF79, and HF93.

\section{Co-occurrence analysis}

Finally, co-occurrence analysis calculates the number of times that cards are paired together, independently of the group in which they are sorted. It aims to identify the relationships between cards (topic), which exists regardless of mental models of the participants. Furthermore, a co-occurrence analysis can be used as a confidence measure to help judge the validity of a card group (Paul, 2014). Thus, the results of co-occurrence analysis helped validate the final human-factor classification proposed. Typically relationships that have $75 \%$ agreement or greater are considered suitable for such analyses (Paul, 2014). Therefore, only those cases in which at least three participants paired the same cards (i.e. $75 \%$ agreement) were considered for the co-occurrence analysis.

\section{4- Hybrid card-sorting case study and analysis of results}

A total of eleven HFs groups were created within the open-card sorting case study. The process outlined in previous section successfully assigned seventy-eight out of the initial ninety-four HFs from the accident database to one of the major category. A new hybrid card-sorting case study was created to allocate the remaining unsorted HFs (i.e. HF7, HF11, HF20, HF28, HF31, HF57, HF59, HF63, HF64, HF65, HF66, HF78, HF86, HF91, HF92, HF94) to one of the eleven categories (Table 2). For this purpose online Optimal Workshop software was used, in which the normalized groups obtained within the open card-sorting case study were provided, but participants were also allowed to create additional groups if they wanted. Hybrid case study included a description of the remaining sixteen HFs.

A statistical analysis was conducted to assess how often a given human factor sorted in the same group. Table 4 provides the results.

Table 4. Statistical analysis. Hybrid card-sorting case study

\begin{tabular}{ccccccccc}
\hline Card & Freq. & Group No & Card & Freq. & Group No & Card & Freq. & Group No \\
\hline 11 & 0.6 & Group 9 & 57 & 0.4 & Group 10 & 91 & 0.4 & Group 3 \\
7 & 0.5 & Group 7 & 59 & 0.4 & Group 8 & 28 & 0.3 & Group 8 \\
20 & 0.5 & Group 8 & 63 & 0.4 & Group 9 & 31 & 0.3 & Group 9 \\
78 & 0.5 & Group 3 & 65 & 0.4 & Group 11 & 64 & 0.3 & Group 11 \\
92 & 0.5 & Group 9 & 66 & 0.4 & Group 6 & & & \\
94 & 0.5 & Group 4 & 86 & 0.4 & Group 10 & & & \\
\hline
\end{tabular}




\section{5- Proposed human-factor classification and comparison with co-occurrence analysis}

On completion of open and hybrid card-sorting case studies, an initial human-factor classification was created. The next step was to review the proposed human-factor grouping by comparing the finally defined classification with the results from the co-occurrence analysis. Here, the cards that had $75 \%$ agreement between participants or greater were compared with the proposed classification to check if paired cards from the co-occurrence analysis were also sorted together in the proposed classification. Table 5provides the agreement between the proposed classification and the co-occurrence results, in which C.A is reference card A, while card B (C.B) is the card that co-occurs i.e. appears in the same group. It can be observed that there is $80 \%$ agreement between the co-occurrence results and the proposed classification prior application of re-definitions in the created groups.

Table 5. Agreement between the co-occurrence analysis and the proposed classification

\begin{tabular}{|c|c|c|c|c|c|c|c|c|c|c|c|}
\hline C. A & C. B & $\begin{array}{l}\text { Proposed } \\
\text { taxonomy }\end{array}$ & C. $\mathbf{A}$ & C. B & $\begin{array}{l}\text { Proposed } \\
\text { taxonomy }\end{array}$ & C. A & C. B & $\begin{array}{l}\text { Proposed } \\
\text { taxonomy }\end{array}$ & C. A & C. B & $\begin{array}{l}\text { Proposed } \\
\text { taxonomy }\end{array}$ \\
\hline 1 & 9 & $\checkmark$ & 14 & 32 & $\checkmark$ & 35 & 61 & $\checkmark$ & 52 & 54 & $\checkmark$ \\
\hline 1 & 10 & $\checkmark$ & 14 & 62 & $\checkmark$ & 36 & 45 & $\checkmark$ & 53 & 61 & $\checkmark$ \\
\hline 1 & 61 & $\checkmark$ & 14 & 67 & $\checkmark$ & 36 & 52 & $\checkmark$ & 54 & 56 & $\checkmark$ \\
\hline 2 & 93 & $\checkmark$ & 14 & 70 & $\checkmark$ & 36 & 69 & $\checkmark$ & 55 & 60 & $\checkmark$ \\
\hline 3 & 32 & $x$ & 14 & 90 & $\checkmark$ & 36 & 81 & $\checkmark$ & 57 & 86 & $x$ \\
\hline 3 & 59 & $x$ & 15 & 36 & $\checkmark$ & 37 & 38 & $x$ & 58 & 64 & $\checkmark$ \\
\hline 5 & 13 & $x$ & 15 & 45 & $\checkmark$ & 41 & 73 & $\checkmark$ & 58 & 71 & $\checkmark$ \\
\hline 5 & 32 & $x$ & 15 & 52 & $\checkmark$ & 42 & 43 & $\checkmark$ & 58 & 76 & $\checkmark$ \\
\hline 5 & 67 & $x$ & 15 & 69 & $\checkmark$ & 42 & 55 & $\checkmark$ & 58 & 89 & $x$ \\
\hline 5 & 70 & $x$ & 15 & 81 & $\checkmark$ & 42 & 60 & $\checkmark$ & 62 & 67 & $\checkmark$ \\
\hline 5 & 90 & $x$ & 16 & 26 & $\checkmark$ & 43 & 60 & $\checkmark$ & 62 & 70 & $\checkmark$ \\
\hline 6 & 48 & $\checkmark$ & 16 & 84 & $\checkmark$ & 44 & 68 & $\checkmark$ & 62 & 90 & $\checkmark$ \\
\hline 6 & 51 & $x$ & 17 & 47 & $\checkmark$ & 45 & 52 & $\checkmark$ & 64 & 65 & $\checkmark$ \\
\hline 6 & 58 & $\checkmark$ & 17 & 49 & $\checkmark$ & 45 & 54 & $\checkmark$ & 64 & 76 & $\checkmark$ \\
\hline 6 & 64 & $\checkmark$ & 18 & 40 & $\checkmark$ & 46 & 83 & $\checkmark$ & 67 & 70 & $\checkmark$ \\
\hline 6 & 71 & $\checkmark$ & 18 & 62 & $\checkmark$ & 46 & 84 & $\checkmark$ & 67 & 90 & $\checkmark$ \\
\hline 6 & 76 & $\checkmark$ & 19 & 46 & $\checkmark$ & 48 & 51 & $x$ & 69 & 81 & $\checkmark$ \\
\hline 6 & 89 & $x$ & 19 & 57 & $x$ & 48 & 58 & $\checkmark$ & 70 & 90 & $\checkmark$ \\
\hline 8 & 72 & $\checkmark$ & 23 & 45 & $\checkmark$ & 48 & 64 & $\checkmark$ & 71 & 76 & $\checkmark$ \\
\hline 9 & 10 & $\checkmark$ & 23 & 52 & $\checkmark$ & 48 & 71 & $\checkmark$ & 71 & 82 & $\checkmark$ \\
\hline 9 & 61 & $\checkmark$ & 23 & 54 & $\checkmark$ & 48 & 76 & $\checkmark$ & 74 & 75 & $\checkmark$ \\
\hline 10 & 61 & $\checkmark$ & 23 & 56 & $\checkmark$ & 48 & 89 & $x$ & 76 & 89 & $x$ \\
\hline 12 & 29 & $\checkmark$ & 30 & 47 & $\checkmark$ & 49 & 66 & $\checkmark$ & 77 & 88 & $\checkmark$ \\
\hline 13 & 67 & $\checkmark$ & 31 & 32 & $\checkmark$ & 50 & 59 & $\checkmark$ & 80 & 87 & $\checkmark$ \\
\hline 13 & 70 & $\checkmark$ & 31 & 86 & $x$ & 51 & 58 & $x$ & 93 & 94 & $x$ \\
\hline 13 & 90 & $\checkmark$ & 32 & 59 & $x$ & 51 & 76 & $x$ & & & \\
\hline 14 & 18 & $\checkmark$ & 32 & 62 & $\checkmark$ & 51 & 89 & $\checkmark$ & & & \\
\hline
\end{tabular}

To further improve the agreement between co-occurrence results and the proposed classification, additional adjustments were applied to the proposed human-factor classification by field experts. HFs that were reallocated within the proposed classification were as follows: HF3, HF5, HF37, HF50, HF51, HF59, HF86, HF89, and HF94. 
Consequently, by comparing the results from the co-occurrence analysis with the re-adjusted classification, an agreement of more than $98 \%$, was achieved.

Later on, expert opinion was sought to analyze the finally proposed classification. The aim was to understand if the initial human factors from the accident database were appropriately allocated in the new classification. Three experts assessed the distribution of human factors in the new classification using a five point Likert scale. As a final adjustment, it was decided to re-allocate fourteen HFs (i.e. HF23, HF28, HF33, HF35, HF41, HF48, HF50, HF54, HF58, HF64, HF65, HF68, HF91, HF94) as suggested by the expert review. Table 6 depicts the HFs' classification proposed after all the analysis carried out in this study. This fulfills the main purpose of this paper i.e. to develop a set of more generic and better-defined human-factors groups by applying a card-sorting technique.

Table 6. Final human-factor classification after incorporating the findings from expert analysis

\begin{tabular}{lll}
\hline No & Group Name & Human Factors \\
\hline 1 & Commercial pressure & 8,39, and 72 \\
2 & Effect of environmental and external factors & $77,80,87$, and 88 \\
3 & Improper design, installation and working environment & $1,9,10,12,28,29,44,50,53,57,61,79$, and 86 \\
4 & Inadequate leadership and supervision & $4,16,19,23,25,26,46,48,58,64,65,83,84$, and 94 \\
5 & Lack of communication and coordination & $42,43,55$, and 60 \\
6 & Lack of, improper or late maintenance & $17,24,30,35,47,49$, and 66 \\
7 & Lack of training & $7,15,27,36,45,52,56,69,81$, and 91 \\
8 & Safety culture & 20 and 33 \\
& Safety management system: Inadequate procedures or & $3,5,11,13,14,18,21,22,31,32,34,37,38,40,59,60$, \\
9 & deviation from Standard Operating Procedure (SOP) & $63,67,70,73,74,75,90$, and 92 \\
10 & Safety management system: Substandard monitoring & $2,41,68,85$, and 93 \\
11 & Unprofessional behaviour & $6,51,54,71,76,79,82$, and 89 \\
\hline
\end{tabular}

\section{Discussion}

In this study, a succinct but generic human-factor classification has been developed by applying card-sorting technique. In order to fulfill the aims of this paper, experts with experience in the fields of accident investigation, HFs and ship operations were invited to participate in the study. Firstly, an open card-sorting technique was used to group the HFs extracted from the historical accident database. Secondly, the results were analyzed qualitatively and quantitatively using statistical and co-occurrence analysis. Hybrid card-sorting method was used to fully achieve the classification of HFs. Finally, expert opinion was employed to analyze the proposed classification, aiming to understand and ensure that the initial HFs from the accident database were appropriately classified.

Open card-sorting analysis resulted in a total of eleven HFs human factors groups, which were able to sort seventyeight of the initial ninety-four HFs human factors from the accident database. Hybrid card-sorting case study was created to allocate the unsorted HFs human factors (i.e. HF7, HF11, HF20, HF28, HF31, HF57, HF59, HF63, HF64, HF65, HF66, HF78, HF86, HF91, HF92, HF94) to one of the eleven categories identified through opencard sorting. Participants were allowed to create additional groups within the hybrid card-sorting session, 
however, only one participant created a new group (i.e. "personal factors") while the remaining participants were preferred to use earlier groups.

Results of open and hybrid card-sorting sessions were qualitatively and quantitatively analyzed. A concern of using qualitative technique (i.e. the group normalization) was the possibility to adding uncertainty to the results as a consequence of using expert knowledge. However, group normalization is one of the most commonly applied qualitative technique to analyze card-sorting. Nevertheless, measures were taken to reduce the uncertainty by selecting experts with significant knowledge on HFs and the MAIB database structure.

In addition, considerations were also made to ensure the reliability and the validity of this study based on the triangulation method that was introduced in the methodology section. Regarding the methodological triangulation, it was ensured that answers were collected using various instruments and similar results were obtained. For example, the first two sets of answers were collected through a workshop, in which participants were assisted to ensure that they understood the meaning of each HF. Thus, two experts were available during the workshop to answer participants' questions regarding the card-sorting method, the HFs from MAIB database and the structure of MAIB database. Moreover, the other two sets of answers were collected directly from the experts of MAIB who were involved in its development and use. Furthermore, to ensure that they commit to the task by providing reliable results, several discussions were held with the experts to explain the objectives and the importance of this study. As a result of the workshop and the online session, similar groups were created by the participants as discussed in the previous sections. In order to complain with the expert triangulation, various experts (i.e. two experts in HFs and one expert in accident investigation) were asked about the suitability of the research methodology, which was designed according to their insights.

Finally, regarding the theoretical triangulation, the results of this study were compared with previous literature, which showed that some of the normalized groups have been also identified as highly important accident contributing factors in past studies. For example, inadequate supervision (group 4, Table-2) has been extensively identified in previous studies as highly related to maritime accidents. For instance, B. M. Batalden and Sydnes (2017) identifying that and unsafe supervision is a main causal factor leading to very serious accidents. B.-M. Batalden and Sydnes (2014) also performed a study to investigate casualties and incidents, revealing that unsafe supervision emerges as the biggest challenge. Furthermore, a study conducted by Macrae (2009) revealed that a lack of supervision and team communication was reported as critical contributing factors. Regarding inadequate or a lack of training (group 7, Table 2), Puisa, Lin, Bolbot, and Vassalos (2018) analyzed numerous maritime accidents, revealing that inadequate training was a frequent causal factor across all reports analyzed. In addition, inadequate procedures (group 9, Table 2) are one of the challenging topics in shipping requiring urgent attention to raise the standards of safety (Kurt, Arslan, Comrie, Khalid, \& Turan, 2016).Furthermore, a study performed by Antão, Almeida, Jacinto, and Guedes Soares (2008) highlighted that inadequate behavior (group 11, Table 2) within particular tasks, led to occupational accidents.

Overall, this study is subject to certain assumptions and practical limitations or challenges. Firstly, the database available for this study includes information about HFs from 129 accidents for the period 2011-2016, as the current taxonomy got introduced in 2011. Hence, in this study, a period of five years could be used. Secondly, due to 
pragmatic reasons, responses from a total of four experts could be collected to sort initial ninety-four HFs from the MAIB database into a more succinct but comprehensive set of HFs categories.

\section{Conclusions}

The goal of this study was to reclassify human factors into smaller and clearer groups, as shortcomings were observed in MAIB database. The purpose was to come up with groups that are broad enough to be used by safety bodies to proactively learn from past experiences and avoid same mistakes. Thus, the results of this study should not be treated as a new taxonomy but a higher level HF categories developed from existing taxonomy which are simpler and self-explanatory.

This study reveals shortcoming of existing classification system, in which high number of HFs are weakly defined, with a big room for misinterpretation. Aforementioned shortcoming not only impacts on the quality of collected data but also makes it difficult to use collected information in future studies. It was identified that some of HF categories are not defined clear enough (e.g. Human Factor No 1. "Anthropometric factors, dimensions", or Human Factor No 6. "Cowboy attitudes, horseplay") and require detailed review of the accident events to understand their meanings. HF No 1 is related to physical dimension problems, for instance a lack of deck space that makes the operations on board more challenging. Whereas, HF No 6 is related to unprofessional behavior, for example the accident description indicated that the officers were holding a party on the bridge. Moreover, within the initial list of ninety-four HFs, it was observed that some of the factors have similar name (e.g. HF No 13. "Emergency plans" and HF No 14. "Emergency procedures", or HF No 36. "Inadequate training program" and HF No 89. "Training ignored"). Thus, the idea of grouping together those factors with similar characteristics appears to be a reasonable alternative and may help researchers to identify potential improvement areas better.

Therefore, our paper is proposing a solution to overcome the shortcoming of existing approach by offering a concise set of HFs categories that can be used by researchers and regulators to focus future improvement affords. These have been developed by incorporating the knowledge of experienced participants in the fields of accident investigations, HFs and ship operations using card-sorting techniques. The card-sorting technique is a wellestablished approach that accounts for the opinions of experts/end-users. Often it is used to explore people's mental models of classifying datasets. Therefore, we believe that it is one of the most suitable technique to be applied for defining the high-level HF categories established in this study.

As recommendations for future work it is suggested a more recent database with larger set of accidents can be used to assess the suitability of HF groups identified in this study. Also, the higher-level HF categories identified in this research study, may be utilized to develop a mechanism for enhancing safety culture in maritime, especially by linking the safety culture dimensions to identified HF categories. Furthermore, as MAIB database follows the EMCIP nomenclature, the study may be repeated if access to such data become available from other European countries to validate the suitability of new groups. Thus, this study can be also repeated by considering data from other boards (e.g. the Transportation Safety Board of Canada, the Australian Transport Safety Bureau, the Accident Investigation Board of Norway, etc.) 


\section{Acknowledgements}

The authors of this paper would like to acknowledge the support of the Marine Accident Investigation Branch (MAIB) who provided the required data for performing this study. In additions, the authors would like to acknowledge the support of Optimal Workshop for granting the use of their card-sorting software.

\section{References}

Antão, P., Almeida, T., Jacinto, C., \& Guedes Soares, C. (2008). Causes of occupational accidents in the fishing sector in Portugal. Safety Science, 46(6), 885-899. doi:https://doi.org/10.1016/j.ssci.2007.11.007

Azadeh, A., \& Zarrin, M. (2016). An intelligent framework for productivity assessment and analysis of human resource from resilience engineering, motivational factors, HSE and ergonomics perspectives. Safety Science, 89, 55-71. doi:http://dx.doi.org/10.1016/j.ssci.2016.06.001

Batalden, B.-M., \& Sydnes, A. K. J. W. J. o. M. A. (2014). Maritime safety and the ISM code: a study of investigated casualties and incidents. 13(1), 3-25. doi:10.1007/s13437-013-0051-8

Batalden, B. M., \& Sydnes, A. K. (2017). What causes 'very serious' maritime accidents? Safety and Reliability - Theory and Applications - epin \& Briš (Eds) (C) 2017 Taylor \& Francis Group, London, ISBN 978-1-138-62937-0.

Bialystok, E., \& Martin, M. M. (2004). Attention and inhibition in bilingual children: Evidence from the dimensional change card sort task. Developmental science, 7(3), 325-339.

Buys, J., \& Clark, J. (1978). Events and causal factors charting. Retrieved from

Bye, R. J., \& Aalberg, A. L. (2018). Maritime navigation accidents and risk indicators: An exploratory statistical analysis using AIS data and accident reports. Reliability Engineering \& System Safety, 176, 174-186. doi:https://doi.org/10.1016/j.ress.2018.03.033

Celik, M., \& Cebi, S. (2009). Analytical HFACS for investigating human errors in shipping accidents. Accident Analysis \& Prevention, 41(1), 66-75.

Chauvin, C., Lardjane, S., Morel, G., Clostermann, J.-P., \& Langard, B. (2013). Human and organisational factors in maritime accidents: Analysis of collisions at sea using the HFACS. Accident Analysis \& Prevention, 59, 26-37. doi:https://doi.org/10.1016/j.aap.2013.05.006

Chen, S.-T., Wall, A., Davies, P., Yang, Z., Wang, J., \& Chou, Y.-H. (2013). A Human and Organisational Factors (HOFs) analysis method for marine casualties using HFACS-Maritime Accidents (HFACS-MA). Safety Science, 60, 105-114. doi:http://dx.doi.org/10.1016/i.ssci.2013.06.009

Deibel, K., Anderson, R., \& Anderson, R. (2005). Using edit distance to analyze card sorts. Expert Systems, 22(3), 129-138.

Duncan, N. (2012). Sexual bullying: Gender conflict and pupil culture in secondary schools: Routledge.

Eliopoulou, E., Papanikolaou, A., \& Voulgarellis, M. (2016). Statistical analysis of ship accidents and review of safety level. Safety Science, 85, 282-292. doi:http://dx.doi.org/10.1016/i.ssci.2016.02.001

EMSA. (2016). EMCIP current taxonomy, glossary of reporting attributes.

Eppler, M. J., \& Platts, K. (2007). An empirical classification of visual methods for management: Results of picture sorting experiments with managers and students. Paper presented at the 200711 th International Conference Information Visualization (IV'07).

Faiks, A., \& Hyland, N. (2000). Gaining user insight: a case study illustrating the card sort technique. College research libraries, 61(4), 349-357.

Fincher, S., \& Tenenberg, J. (2005). Making sense of card sorting data. Expert Systems, 22(3), 89-93.

Flick, U. (2018). Designing qualitative research: Sage.

Fu, G., Cao, J.-L., Zhou, L., \& Xiang, Y.-C. J. P. S. (2017). Comparative study of HFACS and the 24Model accident causation models. 14(3), 570-578. 
Graziano, A., Teixeira, A. P., \& Guedes Soares, C. (2016). Classification of human errors in grounding and collision accidents using the TRACEr taxonomy. Safety Science, 86, 245-257. doi:http://dx.doi.org/10.1016/j.ssci.2016.02.026

Haaland, K. Y., Vranes, L. F., Goodwin, J. S., \& Garry, P. J. (1987). Wisconsin Card Sort Test performance in a healthy elderly population. Journal of Gerontology, 42(3), 345-346.

Hudson, W. (2012). Card sorting. Encyclopedia of human-computer interaction.

Jeong, K., Choi, B., Moon, J., Hyun, D., Lee, J., Kim, I., . . Kang, S. (2016). Risk assessment on abnormal accidents from human errors during decommissioning of nuclear facilities. Annals of Nuclear Energy, 87, Part 2, 1-6. doi:http://dx.doi.org/10.1016/i.anucene.2015.08.009

Kececi, T., \& Arslan, O. (2017). SHARE technique: A novel approach to root cause analysis of ship $\begin{array}{lllll}\text { accidents. Safety } \quad \text { Science, } & \text { 96(Supplement } & \text { C), }\end{array}$ doi:https://doi.org/10.1016/i.ssci.2017.03.002

Kirwan, B. (1996). The validation of three human reliability quantification techniques - THERP, HEART and JHEDI: Part 1 - technique descriptions and validation issues. Applied Ergonomics, 27(6), 359-373. doi:http://dx.doi.org/10.1016/S0003-6870(96)00044-0

Kurt, R. E., Arslan, V., Comrie, E., Khalid, H., \& Turan, O. (2016). SEAHORSE procedure improvement system. Paper presented at the 6th Conference on Design for Safety.

Laws, K. R. (1999). A meta-analytic review of Wisconsin Card Sort studies in schizophrenia: general intellectual deficit in disguise? Cognitive Neuropsychiatry

4(1), 1-30.

Lloyd, D., Dykes, J., \& Radburn, R. (2008). Mediating geovisualization to potential users and prototyping a geovisualization application.

Luo, M., \& Shin, S.-H. (2016). Half-century research developments in maritime accidents: Future directions. Accident Analysis \& Prevention. doi:http://dx.doi.org/10.1016/j.aap.2016.04.010

Macrae, C. (2009). Human factors at sea: common patterns of error in groundings and collisions. Maritime Policy \& Management, 36(1), 21-38.

Navas de Maya, B., Ahn, S. I., \& Kurt, R. E. (2019). Statistical analysis of MAlB database for the period 1990-2016. Paper presented at the International Conference Association of the Mediterranean, Varna, Bulgaria.

Navas de Maya, B., Kurt, R. E., \& Turan, O. (2018). Application of fuzzy cognitive maps to investigate the contributors of maritime collision accidents. Transport Research Arena (TRA) 2018.

Nicholson, A. (2016). What Factors Influence School Choice, with Particular Reference to School Reputation? , Anglia Ruskin University,

Papanikolaou, A., Bitha, K., Eliopoulou, E., Ventikos, N. J. M. T., \& Engineering, L. (2014). Statistical analysis of ship accidents occurred in the period 1990-2012 and assessment of safety level of ship types.

Paul, C. L. (2014). Analyzing card-sorting data using graph visualization. Journal of Usability Studies, 9(3), 87-104.

Puisa, R., Lin, L., Bolbot, V., \& Vassalos, D. (2018). Unravelling causal factors of maritime incidents and accidents. Safety Science, 110, 124-141.

Righi, C., James, J., Beasley, M., Day, D. L., Fox, J. E., Gieber, J., . . Ruby, L. (2013). Card sort analysis best practices. Journal of Usability Studies, 8(3), 69-89.

Rothblum, A. M. (2000). Human error and marine safety. Paper presented at the National Safety Council Congress and Expo, Orlando, FL.

Rugg, G., \& Petre, M. (2007). A Gentle Guide to Research Methods. 1st. In: Maidenhead, Berkshire: Open University Press.

Sanders, K., Fincher, S., Bouvier, D., Lewandowski, G., Morrison, B., Murphy, L., . . Thomas, L. (2005). A multi - institutional, multinational study of programming concepts using card sort data. Expert Systems

22(3), 121-128. 
Schröder-Hinrichs, J. U., Baldauf, M., \& Ghirxi, K. T. (2011). Accident investigation reporting deficiencies related to organizational factors in machinery space fires and explosions. Accident Analysis \& Prevention, 43(3), 1187-1196.

Sinha, R., \& Boutelle, J. (2004). Rapid information architecture prototyping. Paper presented at the Proceedings of the 5th conference on Designing interactive systems: processes, practices, methods, and techniques.

Smith, D., Veitch, B., Khan, F., \& Taylor, R. (2017). Understanding industrial safety: Comparing Fault tree, Bayesian network, and FRAM approaches. Journal of Loss Prevention in the Process Industries, 45, 88-101. doi:http://dx.doi.org/10.1016/i.jp.2016.11.016

Taber, W. (1987). Estimation of expert weights using fuzzy cognitive maps. Paper presented at the Proc. First International Conference on Neural Networks.

Tullis, T. S. (1985). Designing a menu-based interface to an operating system. Paper presented at the ACM SIGCHI Bulletin.

Turan, O., Kurt, R. E., Arslan, V., Silvagni, S., Ducci, M., Liston, P., . . Papadakis, G. (2016). Can We Learn from Aviation: Safety Enhancements in Transport by Achieving Human Orientated Resilient Shipping Environment. Transportation Research Procedia, 14, 1669-1678. doi:http://dx.doi.org/10.1016/i.trpro.2016.05.132

Ung, S.-T. (2015). A weighted CREAM model for maritime human reliability analysis. Safety Science, 72, 144-152. doi:https://doi.org/10.1016/i.ssci.2014.08.012

Ung, S.-T. (2019). Evaluation of human error contribution to oil tanker collision using fault tree analysis and modified fuzzy Bayesian Network based CREAM. Ocean Engineering, 179, 159-172. doi:https://doi.org/10.1016/i.oceaneng.2019.03.031

Ventikos, N., Papanikolaou, A., Louzis, K., \& Koimtzoglou, A. J. O. E. (2018). Statistical analysis and critical review of navigational accidents in adverse weather conditions. 163, 502-517.

Yang, Z. L., Bonsall, S., Wall, A., Wang, J., \& Usman, M. (2013). A modified CREAM to human reliability quantification in marine engineering. Ocean Engineering, 58, 293-303. doi:http://dx.doi.org/10.1016/i.oceaneng.2012.11.003

Yıldırım, U., Başar, E., \& Uğurlu, Ö. (2017). Assessment of collisions and grounding accidents with human factors analysis and classification system (HFACS) and statistical methods. Safety Science. doi:https://doi.org/10.1016/j.ssci.2017.09.022

Zelazo, P. D. (2006). The Dimensional Change Card Sort (DCCS): A method of assessing executive function in children. Nature protocols, 1(1), 297.

Zhang, S., He, W., Chen, D., Chu, J., \& Fan, H. (2019). A dynamic human reliability assessment approach for manned submersibles using PMV-CREAM. International Journal of Naval Architecture and Ocean Engineering, 11(2), 782-795. doi:https://doi.org/10.1016/j.ijnaoe.2019.03.002

Zhou, Q., Wong, Y. D., Xu, H., Thai, V. V., Loh, H. S., \& Yuen, K. F. (2017). An enhanced CREAM with stakeholder-graded protocols for tanker shipping safety application. Safety Science, 95, $140-$ 147. doi:https://doi.org/10.1016/j.ssci.2017.02.014 